\title{
Predicting the Development of Brain Metastases in Patients with Local/Regional Melanoma
}

\author{
TIMOTHY L. FRANKEL, MD, ${ }^{1}$ ZUBIN M. BAMBOAT, MD, ${ }^{1}$ CHARLOTTE ARIYAN, ${ }^{2}{ }^{1}{ }^{1}$ DANIEL COIT, MD, ${ }^{1}$ \\ MICHAEL S. SABEL, MD, ${ }^{2}$ AND MARY S. BRADY, $\mathrm{MD}^{1}{ }^{*}$ \\ ${ }^{1}$ Department of Surgery, Gastric and Mixed Tumor Service, Memorial Sloan-Kettering Cancer Center, New York, New York \\ ${ }^{2}$ Department of Surgery, University of Michigan, Ann Arbor, Michigan
}

Background: The brain is a common site of recurrence in melanoma patients. Brain recurrence may present as a seizure, hemorrhage, or death. We sought to determine predictors of brain metastases in patients with primary and regional melanoma in order to facilitate targeted screening. Methods: Prospectively maintained databases were used to identify patients treated for local or regional melanoma who developed stage IV melanoma with and without brain metastasis at initial recurrence. One hundred twenty patients were identified with brain relapse and compared to 487 patients without brain recurrence.

Results: On univariate analysis, patients with brain metastases were younger (55 vs. 59yrs, $P=0.04$ ) but did not differ in primary site (head and neck $23 \%$ vs. $21 \%, P=0.20$ ). Brain metastasis patients had thinner primaries (mean 3.4 vs. $4.5 \mathrm{~mm}, P=0.01$ ). There were no other pathologic differences including ulceration $(55 \%$ vs. $53 \%, P=0.75)$, mitoses $(7$ vs. $7.5, P=0.61)$ or histologic subtype. Younger age and decreased Breslow thickness were independently associated with brain metastases at stage IV recurrence $(\mathrm{OR}=1.10 P=0.01$ and $\mathrm{OR}=1.02 P=0.02$, respectively).

Conclusions: Our analysis, the largest to date, demonstrates that thinner Breslow depth and younger age were associated with brain recurrence at first presentation with Stage IV disease.

J. Surg. Oncol. 2014;109:770-774 (C) 2014 Wiley Periodicals, Inc.

\section{KEY WoRDs: melanoma; metastases; brain}

\section{INTRODUCTION}

The role of cross-sectional imaging following treatment of clinically localized melanoma, as defined by the National Comprehensive Cancer Network (NCCN) is limited, with routine history and physical exam considered adequate in the absence of symptoms of recurrent disease [1]. Patients with stage III disease are more commonly subjected to routine imaging, particularly during the first 3 years following treatment. While signs and symptoms of visceral recurrence may be subtle, such as vague discomfort, fatigue, or nausea, recurrence in the brain may result in seizure, mental status changes or paralysis [2]. Current surveillance guidelines do not recommend routine brain imaging for asymptomatic patients with stage I-III melanoma $[1,3]$. If a high-risk population could be identified, however, targeted screening could be offered, potentially allowing for diagnosis and treatment prior to development of symptoms.

While no data exists regarding the impact of early detection of brain lesions on survival, prompt treatment with radiotherapy or surgical resection may decrease the likelihood of neurologic catastrophe due to edema, hemorrhage, or herniation $[4,5]$. We identified patients treated for primary and/or regional melanoma who developed brain metastases as part of their first distant recurrence. Baseline demographic characteristics and histopathologic data were used to compare these patients to those who developed distant non-CNS metastases in order to identify predictors of brain metastasis as a component of systemic relapse.

\section{PATIENTS AND METHODS}

Approval for this study was obtained by the Institutional Review Boards (IRB) of Memorial Sloan-Kettering Cancer Center (MSKCC) and the University of Michigan Medical Center (UMMC).

\section{Study Patients}

Prospectively maintained melanoma databases were queried to identify patients who underwent resection of primary or regional cutaneous melanoma at UMMC or MSKCC, and subsequently developed distant metastatic disease during follow-up. Patients under the age of 18 , no evidence of distant metastatic disease, or lost to followup were excluded from analysis. Those who presented with Stage IV disease at the time of their initial diagnosis and those with uveal or mucosal melanoma were also excluded.

\section{Patient and Tumor Characteristics}

Patient characteristics were collected from hospital records and included: gender, age, location of the primary melanoma (head/neck, trunk, lower extremity, and upper extremity) and stage at diagnosis. Histologic features of the primary melanoma were reported by pathologists with extensive experience in dermatopathology and included: Breslow thickness (in millimeters), Clark level, mitotic rate per square millimeter, histologic subtype, ulceration, perineural and lymphovascular invasion, regression, immune infiltrate and satellitosis. Sentinel lymph node (SLN) biopsy was performed, when appropriate, as

Grant sponsor: There were no funding sources for the conduct of this study. The authors report no conflicts of interest in the conduct of this study. *Correspondence to: Mary S. Brady, MD, FACS, 1275 York Ave, New York, NY 10065. Fax: 212-794-5847. E-mail: bradym@mskcc.org

Received 13 November 2013; Accepted 14 January 2014

DOI 10.1002/jso. 23574

Published online 20 February 2014 in Wiley Online Library (wileyonlinelibrary.com) 
previously described [6]. Although there was no absolute standard protocol for post-treatment follow-up, most patients with stage I and II melanoma were examined every 3-6 months. Yearly chest X-rays, LDH levels, liver function tests, and CBCs were obtained when deemed appropriate, but were not routine. Only new symptoms or locoregional recurrence prompted evaluation with cross-sectional imaging using CT scanning, PET/CT scanning, or MRI. Patients who were without evidence of disease following treatment for stage III melanoma were more likely to undergo routine cross-sectional imaging with PET/CT or CT scanning compared to those with stage I or II disease, particularly for the first 2 to 3 years following surgery. Routine CNS imaging was not employed, however, brain imaging (usually MRI) was routinely performed in patients diagnosed with stage IV disease.

\section{Statistical Analysis}

Nominal variables were evaluated using two-tailed $\chi^{2}$ or Fisher's exact tests when appropriate. Continuous variables were assessed with univariate logistic regression for parametric values and Wilcoxon rank sum for non-parametric values. Multivariate logistic regression models were created using all factors with an alpha $<0.10$ on univariate analysis and variables previously identified as associated with brain metastases [7] (head and neck primary and ulceration). Kaplan-Meier survival curves were created to determine differences in time to recurrence and survival. A $P$-value $<0.05$ were considered significant.

\section{RESULTS}

Six hundred seven patients were identified who were treated for stage I-III melanoma and subsequently developed distant metastatic disease during follow-up after treatment for local or regional melanoma at MSKCC or UMMC. There were 415 males and 192 females with a median age of 59 (18-89) years and 53 (20-88) years, respectively. Three-hundred three patients presented with stage III disease (50\%) while the remainder had disease limited to the primary site (stage II$36 \%$, stage $\mathrm{I}-14 \%)$. There were 12 patients (2\%) with unknown primaries, $242(40 \%)$ with lesions located on the trunk, $223(37 \%)$ on an extremity, and $126(21 \%)$ on the head or neck. Breslow thickness ranged from 0.3 to $35 \mathrm{~mm}$ with a mean thickness of $4.3 \mathrm{~mm}$. Fifty-three percent of the primary lesions were ulcerated and $19 \%$ showed evidence of regression. Angiolymphatic invasion was identified in $22 \%$ of patients and $13 \%$ of patients had primaries with perineural infiltration. Primary melanomas were classified as either nodular $(39 \%)$ or superficial spreading melanomas (35\%) with acral (11\%), desmoplastic $(6 \%)$, and lentigo maligna (3\%) comprising the remainder. Mitotic figures were identified in primary tumors from 446 patients with a median number of four (range 0-50).

Because most patients presented with deep primary lesions or occasionally palpable adenopathy, regional spread to lymph nodes was assessed in $93 \%$ of patients. Patients without clinically evident regional disease underwent SLN biopsy (82\%). In addition, 102 patients (18\%) had either an elective or therapeutic regional lymphadenectomy. A median of four lymph nodes were positive in those undergoing any lymphadenectomy (range 1-54).

At the time of analysis, 450 patients $(74 \%)$ were dead of disease and $115(19 \%)$ alive with disease. Thirty-one patients (5\%) underwent metastasectomy or were complete responders to systemic therapy and had no evidence of residual disease at the most recent follow-up. Eleven patients died of "other" or "unknown" causes. The median survival for all patients was 30 months from diagnosis of the primary and 6 months from the discovery of metastatic disease.

Of the 607 patients, 120 patients $(20 \%)$ had brain metastases as part of their initial distant recurrence. Of the 120 patients, the brain was the sole site of recurrence in 63 patients (53\% of the brain metastasis cohort).
For those with synchronous recurrence (57 patients), other sites of disease included the lung (58\%), liver (33\%), and bone (13\%). This did not differ significantly from those with extra-cranial recurrence, so that there was nothing "unique" about the pattern of synchronous metastasis occurring with brain relapse.

To identify factors predictive of CNS failure patients with brain metastases were compared to those with extra-cranial recurrence (487 patients). Demographic characteristics for patients with and without brain metastases are listed in Table I. There was a similar distribution of gender with $73 \%$ and $67 \%$ males in the brain metastasis and non-CNS metastasis, respectively $(P=0.32)$. Location of the primary lesion did not differ between groups, with a comparable frequency of head and neck melanoma (23\% and $21 \%$, respectively). Patients with brain metastases tended to be younger with a median age of 55 years compared to 59 years for those with non-brain metastases $(P=0.04)$. The distribution of stage at presentation was similar between groups with a majority of patients with and without brain metastases initially treated with stage III disease (44\% and $49 \%$, respectively).

Histopathologic features of the primary lesion are presented in Table II. The primary tumors of patients who developed brain metastases were significantly thinner, with a mean Breslow thickness of $3.43 \mathrm{~mm}$, compared to $4.53 \mathrm{~mm}$ in patients with no brain metastasis $(P=0.01)$. There was, however no difference in the Clark level, ulceration, lymphovascular invasion, regression, or lymphocytic infiltrate between the two groups. On univariate analysis, perineural invasion was encountered more frequently in the patients with distant extra-cranial metastases (15\%) compared to those with brain metastasis $(7 \%$; $P=0.05)$. The distribution of histologic subtype was similar between groups with a slight trend towards more superficial spreading melanoma in patients with brain relapse.

Antecedent SLN biopsy was more common in patients who developed brain metastases. There was however, no difference in the incidence of SLN positivity or total number of positive SLNs. One hundred four patients who relapsed in the brain had undergone SLN biopsy, of these $53 \%$ were found to be SLN negative $(53 \%)$. One hundred eighty of 360 patients who developed stage IV disease without brain relapse had undergone SLN mapping, of whom $50 \%(\mathrm{n}=180)$ had $(+)$ SLNs $(P=\mathrm{NS})$. When including patients who underwent lymphadenectomy without sentinel node biopsy, there were no differences in total number of positive lymph nodes between the two groups.

A multivariate logistic regression model was constructed using variables with $P<0.1$ including, Breslow thickness, age, SLN biopsy,

TABLE I. Clinical Features of Patients Treated for Stage I-III Melanoma Who Developed Distant Metastasis with $(n=120)$ or Without $(n=487)$ Brain Recurrence as a Component of Relapse

\begin{tabular}{lccr}
\hline & $\begin{array}{c}\text { Brain mets } \\
(\mathrm{n}=120)\end{array}$ & $\begin{array}{c}\text { No brain mets } \\
(\mathrm{n}=487)\end{array}$ & $P$-value \\
\hline Gender & & & 0.32 \\
Male & $73 \%$ & $67 \%$ & \\
Female & $28 \%$ & $33 \%$ & 0.04 \\
Age (median) & 55 yrs $(20-88$ yrs $)$ & 59 yrs $(18-89$ yrs $)$ & 0.20 \\
Primary Site & & & \\
Head and Neck & $23 \%$ & $21 \%$ & \\
Extremity & $29 \%$ & $39 \%$ & 0.31 \\
Trunk & $47 \%$ & $38 \%$ & \\
Unk primary & $2 \%$ & $2 \%$ & \\
Stage at Diagnosis & & & \\
I & $19 \%$ & $13 \%$ & \\
II & $37 \%$ & $37 \%$ & \\
III & $44 \%$ & $49 \%$ & \\
\hline
\end{tabular}

Mets, metastasis. 
TABLE II. Histologic Features of Primary Lesions from Patients Treated for Stage I-III Melanoma Who Developed Distant Metastasis with $(n=120)$ or Without $(n=487)$ Brain Recurrence as a Component of Relapse

\begin{tabular}{|c|c|c|c|}
\hline & $\begin{array}{l}\text { With brain mets } \\
\qquad(\mathrm{n}=120)\end{array}$ & $\begin{array}{l}\text { No brain mets } \\
\quad(\mathrm{n}=487)\end{array}$ & $P$-value \\
\hline Thickness (mean) & $3.43 \mathrm{~mm}$ & $4.53 \mathrm{~mm}$ & 0.01 \\
\hline Clark level & & & 0.20 \\
\hline 2 & 0 & $2 \%$ & \\
\hline 3 & $5 \%$ & $7 \%$ & \\
\hline 4 & $83 \%$ & $73 \%$ & \\
\hline 5 & $12 \%$ & $18 \%$ & \\
\hline Ulceration & & & 0.75 \\
\hline Yes & $55 \%$ & $53 \%$ & \\
\hline No & $45 \%$ & $47 \%$ & \\
\hline Host response & & & 0.50 \\
\hline None & $84 \%$ & $87 \%$ & \\
\hline Slight & $1 \%$ & $2 \%$ & \\
\hline Brisk & $15 \%$ & $11 \%$ & \\
\hline Regression & & & 0.79 \\
\hline Yes & $18 \%$ & $20 \%$ & \\
\hline No & $82 \%$ & $80 \%$ & \\
\hline Lymphovasc inv & & & 0.79 \\
\hline Yes & $23 \%$ & $21 \%$ & \\
\hline No & $77 \%$ & $79 \%$ & \\
\hline Perineural inv & & & 0.05 \\
\hline Yes & $6 \%$ & $15 \%$ & \\
\hline No & $94 \%$ & $85 \%$ & \\
\hline Satellitosis & & & 0.81 \\
\hline Yes & $13 \%$ & $12 \%$ & \\
\hline No & $87 \%$ & $88 \%$ & \\
\hline Mitosis (mean) & 7.02 & 7.45 & 0.61 \\
\hline Histology & & & 0.39 \\
\hline Superficial spr & $45 \%$ & $33 \%$ & \\
\hline Nodular & $35 \%$ & $39 \%$ & \\
\hline Acral & $11 \%$ & $11 \%$ & \\
\hline Desmoplatic & $4 \%$ & $6 \%$ & \\
\hline Lentigo malig & $1 \%$ & $4 \%$ & \\
\hline Other & $5 \%$ & $6 \%$ & \\
\hline
\end{tabular}

Mets, metastasis; inv, invasion; spr, spreading; malig, malignant.

and perineural invasion (Table III). Also included were variables previously reported as associated with brain metastases including ulceration [2,7] and anatomic location of the primary lesion [8]. On multivariate analysis advanced age was protective of brain metastases development with an odds ratio of 0.9 per year $(P=0.01)$. Thickness of the primary lesion was inversely proportional to CNS failure with an odds ratio of 0.98 per $\mathrm{mm}$ of depth.

Kaplan-Meier survival curves were created to determine differences in overall survival and time to recurrence between patients with and without brain metastases as a component of initial distant recurrence (Figs. 1 and 2). While there were no differences in median time to recurrence (19 months for each group, Fig. $1, P=0.29$ ), the median survival following diagnosis was significantly shorter (6 months) in those with CNS metastases compared to those with only extra-cranial distant metastasis (10 months, $P<0.001$, Fig. 2). Both ulceration and lymph node positivity decreased the time to distant metastasis both in the brain and elsewhere, but had no effect on post-metastatic survival (data not shown).

\section{DISCUSSION}

Melanoma represents the third most common metastatic tumor to the brain, affecting up to $73 \%$ of patients with stage IV disease at autopsy [9]. Adjusting for incidence, melanoma has the greatest propensity for CNS spread of any primary malignancy $[2,10]$. The exact pathophysiology behind this is currently unknown and the source of
TABLE III. A Multivariate Analysis of Factors Previously Reported as Associated with Brain Metastases

\begin{tabular}{lcc}
\hline & Odds ratio & $P$-value \\
\hline Age (per year) & 0.98 & 0.01 \\
Thickness (per mm) & 0.90 & 0.02 \\
Site & 1 & 0.27 \\
$\quad$ Trunk (referent) & 0.97 & \\
Head and neck & 0.64 & \\
$\quad$ Extremity & 1.14 & 0.65 \\
Ulceration & 1.66 & 0.19 \\
Perineural inv & 1.11 & 0.73 \\
SLN biopsy &
\end{tabular}

Young age at initial diagnosis and decreased thickness of the primary lesion were significantly associated with brain relapse when compared to patients who relapse without brain recurrence

Inv, invasion; SLN, sentinel lymph node.

active investigation [11-13]. Isolated brain metastases can often be treated with surgical resection or radiation, with only occasional cures [14-16]. More commonly, patients present with multiple brain metastases and are offered corticosteroids and/or radiation for symptom control. While no data exists regarding the impact of early detection of brain lesions on survival, prompt treatment with radiotherapy or surgical resection may decrease the likelihood of neurologic catastrophe due to edema, hemorrhage, or herniation.

There have been multiple prior studies aimed at identifying predictors of brain metastases in patients with early stage melanoma. Sampson et al. [2] reported on nearly 7,000 patients treated at a single institution for primary melanoma over two decades. During follow-up, $702(10 \%)$ patients developed clinical or radiographic evidence of brain metastases. These patients were compared to all other patients in their melanoma database to identify predictors of brain recurrence. Included in this comparison cohort were patients with early disease (stage 1 and 2) who were treated with surgical resection alone and never recurred. Univariate analysis identified young age, male gender, trunk location, depth of invasion, nodular subtype, and ulceration as predictors of development of brain metastases. Multivariate analysis was not performed. A similar study by Zakrzewski et al. [7] reported on 900 patients with melanoma

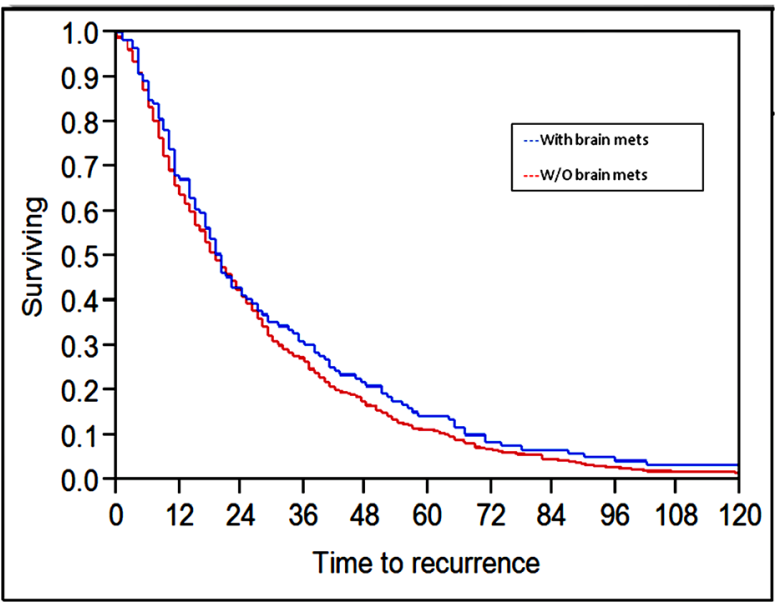

Fig. 1. Kaplan-Meier survival curve illustrating time to recurrence (months) between patients initially treated for stage I-III melanoma who developed distant metastasis with (top curve) and without brain metastases (lower curve) as a component of their initial recurrence. There was no difference in time to presentation with stage IV disease between the two groups $(P=0.29)$. 


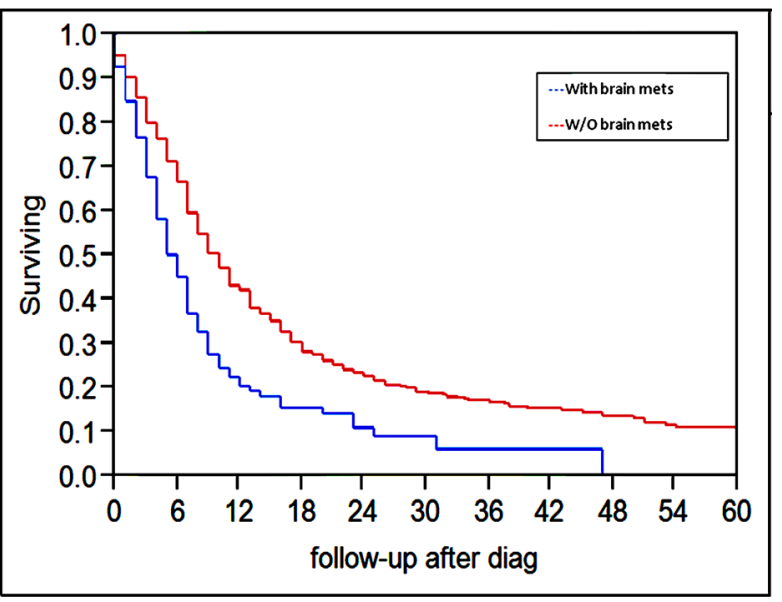

Fig. 2. Kaplan-Meier survival curve illustrating overall survival (months) between patients initially treated for stage I-III melanoma who developed distant metastasis with (lower curve) and without (top curve) brain metastases as a component of their initial recurrence. Not surprisingly, patients with brain metastasis as a component of their presentation with stage IV disease had less favorable overall survival $(P<0.0001)$.

enrolled in a prospective observational database. Demographic data and pathologic characteristics of the primary lesion in patients who presented with or developed brain metastases were compared to the remaining cohort. Again, in this study the comparison cohort included many patients treated for local disease only who never recurred. This is highlighted by the fact that $76 \%$ of patients in the non-brain metastases group had either stage 1 or 2 disease and the overall survival in the cohort was $90 \%$. On multivariate analysis, the authors identified ulceration as a strong predictor of brain metastases with a threefold increased risk of CNS spread. Another predictor was head and neck location of the primary which, when compared to trunk or extremity, was 2.5 -fold more frequent in those with brain relapse. Histologic subtype and Breslow depth showed no association on multivariate analysis with CNS spread. While these prior studies represent the largest and most comprehensive to date, their use of patients with early stage melanoma who never recurred following local resection as comparators makes interpretation of results difficult. The predictors identified may simply represent risk factors for the development of metastatic disease.

To remedy this, we chose to compare patients who were treated for stage I-III melanoma and subsequently recurred in the brain to those who developed distant spread to extracranial sites. Both cohorts include only stage IV patients thereby eliminating the potential bias towards overall predictors of metastasis formation. Similar to results found on univariate analysis by Sampson et al. [2], patients with brain metastases were younger and had thinner lesions than the extracranial metastatic cohort. This may suggest a molecular or genetic phenotype which disseminates hematogenously at an earlier time point. Data supporting this are the increased expression of matrix metalloproteinases (MMP) in melanomas associated with greater vascular invasion and possibly early hematogenous spread [17]. MMPs have also been implicated in brain metastases in lung [18] and breast cancer [19] suggesting a possible link between increased MMP expression and brain metastasis. A study be Xie et al. [20] found that activation of signal transducer and activator of transcription 3 (Stat3) in melanoma leads to increased MMP production, cellular invasion, and brain metastasis in-vivo.

Other pathologic variables such as regression, lymphocytic infiltrate, and mitoses per high powered field were not different between groups and failed to predict brain relapse. Contrary to prior reports [2,7], there was no association between ulceration and CNS spread with equal incidence in both groups. This suggests that the significance of ulceration seen in prior studies may have been due to the use of patients with early stage melanoma who never recur as the comparison group, as previously stated. Although on univariate analysis, it appeared that primary tumors of patients with extra-cranial metastases had a higher incidence of perineural invasion, this failed to prove significant on multivariate analysis. The location of the primary tumors was similar between groups with a trend towards more trunk and fewer extremity lesions in those with brain metastases. There was no difference in the presence of head and neck primaries, contrary to prior studies [7].

Patients with brain metastases were more likely to have a sentinel node biopsy performed although this proved not significant on multivariate analysis. There was no difference in sentinel node positivity or total number of involved lymph nodes between groups. This, along with the similarities in lymphovascular invasion of the primary tumors, suggests no association between regional lymph node involvement and the development of brain metastases in patients destined to develop stage IV melanoma.

While identifying high risk patients could allow for improved screening with neuroimaging, we were unable to find useful clinical or pathologic predictors of brain metastases in early stage patients. Although these patients tended to be younger and have thinner primary lesions, these findings are not useful in identifying a high-risk population. Further research is needed to identify novel biomarkers for neurotropism which may better predict brain metastases in patients with melanoma. This should alert the clinician to remain vigilant, however, in patients with a history of melanoma and subtle symptoms of neurologic concern to consider neuroimaging even in the patient with a negative SLN biopsy as just over half of the patients with brain relapse in our study had negative regional lymph nodes.

\section{REFERENCES}

1. Coit DG, Andtbacka R, Anker CJ, et al.: Melanoma. J Natl Compr Canc Netw 2012;10:366-400.

2. Sampson JH, Carter JH Jr, Friedman AH, et al.: Demographics, prognosis, and therapy in 702 patients with brain metastases from malignant melanoma. J Neurosurg 1998;88:11-20.

3. Shumate CR, Urist MM, Maddox WA: Melanoma recurrence surveillance. Patient or physician based? Ann Surg 1995;221:566569; discussion 569-571.

4. Nguyen TD, DeAngelis LM: Brain metastases. Neurol Clin 2007;25:1173-1192,x-xi.

5. Alexander E 3rd, Moriarty TM, Davis RB, et al.: Stereotactic radiosurgery for the definitive, noninvasive treatment of brain metastases. J Natl Cancer Inst 1995;87:34-40.

6. Morton DL, Wen DR, Wong JH, et al.: Technical details of intraoperative lymphatic mapping for early stage melanoma. Arch Surg 1992;127:392-399.

7. Zakrzewski J, Geraghty LN, Rose AE, et al.: Clinical variables and primary tumor characteristics predictive of the development of melanoma brain metastases and post-brain metastases survival. Cancer 2011;117:1711-1720.

8. Zartman GM, Thomas MR, Robinson WA: Metastatic disease in patients with newly diagnosed malignant melanoma. J Surg Oncol 1987;35:163-164.

9. Harrison BE, Johnson JL, Clough RW, et al.: Selection of patients with melanoma brain metastases for aggressive treatment. Am J Clin Oncol 2003;26:354-357.

10. de la Monte SM, Moore GW, Hutchins GM: Patterned distribution of metastases from malignant melanoma in humans. Cancer Res 1983;43:3427-3433.

11. Vogelbaum MA, Masaryk T, Mazzone P, et al.: S100beta as a predictor of brain metastases: Brain versus cerebrovascular damage. Cancer 2005;104:817-824.

12. Madajewicz S, Karakousis C, West CR, et al.: Malignant melanoma brain metastases. Review of Roswell Park Memorial Institute experience. Cancer 1984;53:2550-2552. 
13. Fidler IJ, Schackert G, Zhang RD, et al.: The biology of melanoma brain metastasis. Cancer Metastasis Rev 1999;18:387400.

14. Wronski M, Arbit E: Surgical treatment of brain metastases from melanoma: A retrospective study of 91 patients. J Neurosurg 2000;93:9-18.

15. Hagen NA, Cirrincione C, Thaler HT, et al.: The role of radiation therapy following resection of single brain metastasis from melanoma. Neurology 1990;40:158-160.

16. Douglas JG, Margolin K: The treatment of brain metastases from malignant melanoma. Semin Oncol 2002;29:518-524.
17. Vihinen P, Koskivuo I, Syrjanen K, et al.: Serum matrix metalloproteinase- 8 is associated with ulceration and vascular invasion of malignant melanoma. Melanoma Res 2008;18:268-273.

18. Yoshida S, Takahashi $\mathrm{H}$ : Expression of extracellular matrix molecules in brain metastasis. J Surg Oncol 2009;100:65-68.

19. Stark AM, Anuszkiewicz B, Mentlein R, et al.: Differential expression of matrix metalloproteinases in brain- and bone-seeking clones of metastatic MDA-MB-231 breast cancer cells. J Neurooncol 2007;81:39-48.

20. Xie TX, Huang FJ, Aldape KD, et al.: Activation of stat 3 in human melanoma promotes brain metastasis. Cancer Res 2006;66:3188-3196. 